\title{
The growing problem of humanizing robots
}

\begin{abstract}
The humanization of robots or, the representation of robots as humans, has contributed to the successful adoption of robots into our society. However, there are also drawbacks to the humanization of robots. Unfortunately, these drawbacks have received much less attention. It may now be time to consider these drawbacks. This article attempts to draw attention to such problems. The article concludes by not only recommending that scholars consider such problems but also consider, in some cases, the possibility of intentionally undermining the humanization of robots.
\end{abstract}

Keywords: robots, humanization of robots, sex robots, robot coworkers, robot friends, love and robots, robot lovers, humanizing robots, robot health care assistants, robot companions
Volume 3 Issue I - 2017

\author{
Lionel P Robert \\ School of Information, University of Michigan, USA
}

Correspondence: Lionel P Robert, School of Information, University of Michigan, USA, Email lprobert@umich.edu

Received: July 10, 2017 | Published: August 29, 2017

\section{Introduction}

One thing that seems to unite many scholars that study robots is the goal of one day creating a fully autonomous human-like robot capable of mimicking all human behaviors and emotions. Advances in artificial intelligence and robotics are beginning to make what was once a thing of science fiction seem not only possible, but probable. To be fair, research has already demonstrated that humans do not need much in the way of prompting to treat and interact with technology as if it was human. ${ }^{1}$ Yet, the current trend toward designing robots to be personal assistants, companions and even lovers may have unintended consequences that we have not fully considered.

\section{Humanizing robots}

In this paper, the humanization of robots is defined as the representation of robots as humans and/or to attribute human like qualities to robots. There are two ways robots are being humanized in our society. The first is through their physical appearance and interactions. This includes physical features added to make robots appear and sound more human. This also includes programs designed to give robots human personality which allows them to behave like humans. The second is through their roles in our society. Roles as co-workers, friends and lovers invoke emotional dependence that has often been associated with humans. As robots increasingly take on these roles, humans are less likely to view co-workers as being nonhuman and more likely to view robots who are co-workers as human.

\section{Benefits of humanizing robots}

To be clear, there have always been benefits to humanizing technology. Research has shown that humans are much more likely to trust robots they view as more human. ${ }^{2,3}$ Convincing humans to trust robots is still an important barrier to the adoption of robots in many areas of society. ${ }^{4}$ However, there are also drawbacks to the humanization of robots. Unfortunately, these drawbacks have received much less attention. This is almost certainly due to our preoccupation with the challenges associated with promoting the adoption of robots. Yet, it may now be time to consider some of the problems with humanizing robots.

\section{The problems with humanizing robots}

The problem with humanizing robots can be organized around three basic premises:

a. Humanization impacts our emotions toward robots. Our emotions toward robots are likely to range from feelings like "fondness" when robots are less humanized to deeper and stronger emotions such as "love" when robots are more humanized.

b. Humanization impacts our expectations of robots. The more humanized robots are the more we expect them to take on human responsibilities in our society.

c. Humanization impacts our expectations of how others should interact with robots. Our view of what is or what is not appropriate use of robots varies by the extent to which we view them as humans.

All three of these premises have important implications for our society discussed below:

\section{Robots as co-workers and friends}

Examples of the Implications of Premise 1: Can the bond between humans and robots be too strong? Robots are often deployed in place of humans to perform dangerous tasks. ${ }^{5}$ As robots become humanized, will humans be less willing to sacrifice their robot co-workers. Evidence of this has already been found in military bomb disposal teams. A recent study found that the more soldiers were attached to their military bomb disposal robot the less willing they were to send it in harm's way. ${ }^{6}$ This of course undermines the robots intended use which is to minimize the loss of human life. Other questions remain, such as: What are the potential long term emotional impacts of intentionally sending your robot co-worker to their death? Should we expect humans to feel a similar sense of loss and guilt when losing a human worker? Robots are also being designed as personal home health care assistants, companions and friends. As mentioned earlier, there are clear benefits to designing such robots to be as human-like as possible. But what are the long term ramifications of such relationships for humans? Are these potentially healthy relationships or inherently problematic and representative of human behavior detrimental to that 
individual and society as a whole? In other words, do we know if such relationships are likely to be more harmful than helpful? Answers to such questions would seem to be a vital prerequisite to determining how human-like robots should be or what, if any, roles should be off limits to robots.

\section{Robots as citizens}

Examples of the Implications of Premise 2: The more humanized robots become the more we might expect them to assume human responsibilities in our society. Recently, Bill Gates proposed a temporary robot tax for companies that employ them to replace human workers. ${ }^{7}$ Although this idea was quickly dismissed, consider another alternative. Imagine taxing robots permanently in much the same way we do humans. This idea seems far-fetched now because many of the robots replacing humans come in the physical form of faceless machines with robotic arms. But, imagine looking across a manufacturing plant and seeing hundreds of fully autonomous humanlike robots at work stations once occupied by human employees. Picturing manufacturing plants teaming with such robots may make the idea of taxing robots seem much more plausible to the average tax payer. The idea of taxing a non-human entity is not itself new. For example, corporations are artificial entities that legally exist separate from the individuals who own or run them. Corporations pay taxes over and above those paid by individuals own or run them.

\section{Robots as lovers}

Examples of the Implications of Premise 3: As these robots become more human-like do we began to view them differently with regards to their use as sexual partners in our society? As robots designed to be sexual partners become more humanized, do we begin to view them less as sexual devices and more as sex slaves? Before this idea is dismissed consider the recent case of an individual who was arrested because the sex robot they ordered resembled a child. ${ }^{8}$ Canadian courts are now in the process of attempting to determine whether or not such an activity is illegal. What is clear is that the mere physical appearance of a robot's changes our expectations about what is appropriate and what is inappropriate behavior with them.

\section{Conclusion}

Although the humanization of robots helps to overcome barriers to adoption it also presents challenges. Scholars have been too preoccupied with overcoming these challenges to consider the problems associated with the humanization of robots. This article attempts to draw attention to such problems. This article will conclude by recommending that scholars not only consider these problems but also consider the possibility of hindering the humanization of robots in some cases.

\section{Acknowledgments}

No financial interest or any conflict of interest exists.

\section{Conflict of interest}

Author declares that there is none of the conflicts.

\section{References}

1. Reeves B, Nass C. How people treat computers, television, and new media like real people and places. UK: CSLI Publications and Cambridge University Press. 1996. p. 3-18.

2. You S, Robert LP. Emotional Attachment, Performance, and Viability in Teams Collaborating with Embodied Physical Action (EPA) Robots. Journal of the Association for Information Systems. 2017.

3. Robert LP, You S. Subgroup Formation in Teams Working with Robots in the Extended Abstracts of the $33^{\text {rd }}$ ACM Conference on Human Factors in Computing Systems. 2015. p. 2097-2102.

4. Creech C, Jayaraman SK, Robert LP, et al. Trust and Control in Autonomous Vehicle Interactions, to be presented at the Morality and Social Trust in Autonomous Robots workshop at the 2017 Robotics: Science and Systems (RSS 2017). UK: Cambridge university. 2017.

5. Robert LP, You S. Human-Robot Interaction in Groups: Theory, Method, and Design for Robots in Groups. GROUP '14 Proceedings of the $18^{\text {th }}$ International Conference on Supporting Group Work. USA. 2014. p. 310-312.

6. Carpenter J. Just doesn't look right: exploring the impact of humanoid robot integration into explosive ordnance disposal teams. Robotics: Concepts, Methodologies, Tools, and Applications IGI Global. 2014. p. $1354-1380$

7. Delaney KJ. The robot that takes your job should pay taxes, says Bill Gates, Quartz Media. 2017.

8. Wakefield J. Call for a ban on child sex robots. 2017. 